\title{
Studies on ovarian quiescence in the lactating bonnet monkey (Macaca radiata)
}

\author{
N. R. MOUDGAL*, R. MANECKJEE, B. R. SRINATH and \\ K. RAMASHARMA \\ Center for Advanced Research in Reproductive Biology, Department of Biochemistry, Indian \\ Institute of Science, Bangalore 560012 , India
}

MS Received 25 January 1985

\begin{abstract}
Lactating bonnet monkeys were used as a model to understand the mechanism of ovarian quiescence during lactation. The ovary of the bonnet monkey in the 3rd month of lactation responds well to exogenous pregnant mare serum gonadotropin stimulation with serum estrogen values reaching maximal levels by day 3 of the gonadotropin injection. The adminstration of ovine prolactin to such monkeys significantly inhibited the ovarian responsiveness to exogenous gonadotropin. The responsiveness of the pituitary of the lactating monkey (in the 3rd month of lactation) to luteinizing hormone releasing hormone injection was suppressed and supplementation with exogenous prolactin further accentuating this effect. The relative ability of chlorpromazine given intravenously/intramuscularly/intranasally to enhance endogenous prolactin levels was assessed. During the first 5 months of lactation when the basal prolactin levels were high, the luteinizing hormone levels were low. As the suckling stimulus reduces and prolactin levels fall, luteinizing hormone levels increase, the first post-parturient mensus occurring by $218 \pm 4$ days. This event was postponed by 3 months on increasing endogenous prolactin levels by administering chlorpromazine $(250 \mu \mathrm{g}$ /day by intranasal mode) over a 5 day period every month starting from the $3 \mathrm{rd}$ month of lactation.
\end{abstract}

Keywords. Lactational amennorrhea; hyperprolactinemia; ovary; luteinizing hormone; lactogenic hormone.

\section{Introduction}

The mechanism whereby ovarian quiescence occurs during lactation is still not well understood. The data largely obtained using the lactating rat as the experimental model suggested that high levels of prolactin seen during lactation must be affecting the normal functioning of the hypothalamo-pituitary axis (Muralidhar et al., 1977; Maneckjee et al., 1976) as well as the responsiveness of the target tissue to gonadotropins (Maneckjee and Moudgal, 1975; Maneckjee et al., 1977) by mechanisms presently unknown. In the lactating rat the level of circulating prolactin could easily be regulated by reducing or increasing the number of pups suckling indicating that the

\footnotetext{
* To whom reprint requests should be addressed.

Abbreviations used: LH, Luteinizing hormone; RIA, radioimmunoassay; s.c., subcutaneous; PMSG, pregnant mare serum gonadotropin; IU, international units; i.m. intramuscular; LHRH, luteinizing hormone releasing hormone; i.v., intravenously.
} 
intensity of suckling is directly correlated to circulating prolactin levels (Amenomori et al., 1970; Trekle et al., 1972). Since this was not feasible in the lactating monkey, recourse to the use of drugs like chlorpromazine which increased prolactin level has been taken. The results presented herein essentially reviews our earlier work in the lactating monkey and describes some of the newer information obtained on the effect of increasing endogenous circulating prolactin level by drug therapy on ovarian function in the lactating monkey.

\section{Materials and methods}

Information on the essential reproductive cycle of the female bonnet monkey (Murty et al., 1980) and their husbandry (Srinath, 1980) in the laboratory has been provided earlier. In the bonnet monkey lactation normally lasts for 6 months as by this time the baby is beginning to partake more and more of the solid food provided to the mother. Lactating mothers are individually caged with their babies and the babies are separated from the mothers only after the occurrance of the first post-parturient menses and this occurs by $218 \pm 4$ days of lactation.

The estrogen and luteinizing hormone (LH) levels in the serum were monitored using radioimmunoassay (RIA) methods standardized in this laboratory earlier by Mukku and Moudgal (1975) and Ramasharma et al. (1978) respectively. The serum prolactin levels were measured using the human prolactin RIA kit kindly provided by NIAMDD, Bethesda, Maryland, USA.

The drug chlorpromazine was administered using the nasal spray mode in $10 \mathrm{~s}$ sprays up each nostril of unanesthetized monkeys according to the procedure described by Anand Kumar et al. (1977). The drug was given dissolved in a vehicle made up of ethanol: propylene glycol: water (3:3:4). Control monkeys, received vehicle only.

\section{Results and dissussion}

\section{Ovarian responsiveness of lactating bonnet monkeys to exogenous gonadotropins}

Lactating bonnet monkeys caged individually with their babies were given in the 3rd month of lactation a daily subcutaneous (s.c.) injection of pregnant mare serum gonadotropin (PMSG) in saline according to the following schedule: day 1-250 international units (IU); day 2-200 IU; day 3-150 IU; days 4 and 5-100 IU and days 6, 7 and 8-75 IU. The choice of monkeys in the 3rd month of lactation was based on our earlier laparoscopic examination of such monkeys which indicated that the ovaries at this time do not contain discernible developing follicles or corpus luteum. While one group of 4 monkeys received the above regimen a second group of 4 monkeys received in addition to PMSG a daily intramuscular (i.m.) injection of $2 \mathrm{mg}$ of ovine prolactin (NIH preparation) given $1 \mathrm{~h}$ earlier to PMSG. Blood samples were removed from femoral venepuncture at time 0 and $24 \mathrm{~h}$ after each PMSG injection for estrogen estimation in serum by appropriate RIA standardized in this laboratory (Mukku and Moudgal, 1975).

The results (figure 1) indicate that the ovary of the monkey even during the active 


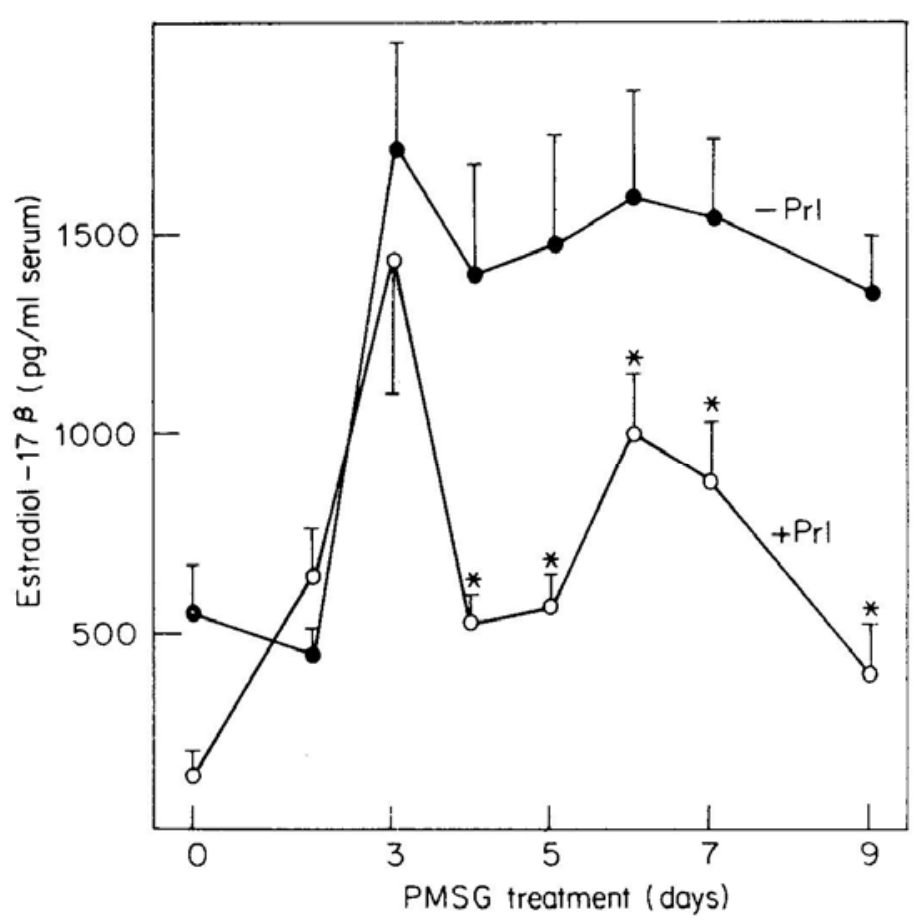

Figure 1. Ovarian responsiveness of monkeys in the 3rd month of lactation to PMSG injection and the effect of prolactin on this. Details of the PMSG and prolactin dose given are provided in the text. $\mathrm{n}$ of each group was 4 . (•), No prolactin; (०), with prolactin.

*Significantly different from the corresponding controls $P<0 \cdot 01$ (after Maneckjee et al., 1977).

phase of lactation was able to respond to exogenous gonadotropin by producing significant elevations in estrogen levels. Interestingly, this ability of PMSG to evoke a response was markedly reduced by administering lactating monkeys an additional dose of $2 \mathrm{mg}$ of ovine prolactin/day. The inhibition in response was significant from day 4 of PMSG treatment $(P<0 \cdot 01)$. The mechanism by which prolactin inhibited ovarian response is presently unclear.

Responsiveness of the pituitary of lactating monkeys to exogenous luteimizing hormone releasing hormone

We had earlier observed (Maneckjee et al., 1976) that the efficacy of exogenous luteinizing hormone releasing hormone (LHRH) (100 $\mu \mathrm{g}$ AY-24, 031-4 gifted by Dr. F. Labrie) given intraveneously (i.v.) dissolved in $1 \mathrm{ml}$ of $0.9 \% \mathrm{NaCl}$, to stimulate secretion of LH was significantly effected if the lactating monkey was actively suckling the baby. All monkeys used in this experiment were in the 2 nd month of lactation. Unlike in the actively suckling group where the baby was left with the mother, in the control group the baby was removed from the mother for $24 \mathrm{~h}$ before injecting LHRH. Thus in the control group (figure 2B) the LH levels in serum increased significantly by 10 min but returned to basal values by $20 \mathrm{~min}$. In contrast, in addition to the actively suckling group (figure 2A) having a lowered basal LH levels (control: $0 \cdot 95 \pm \cdot 0 \cdot 7 \mathrm{ng} \mathrm{hLH} / \mathrm{ml} v \mathrm{~s}$ 


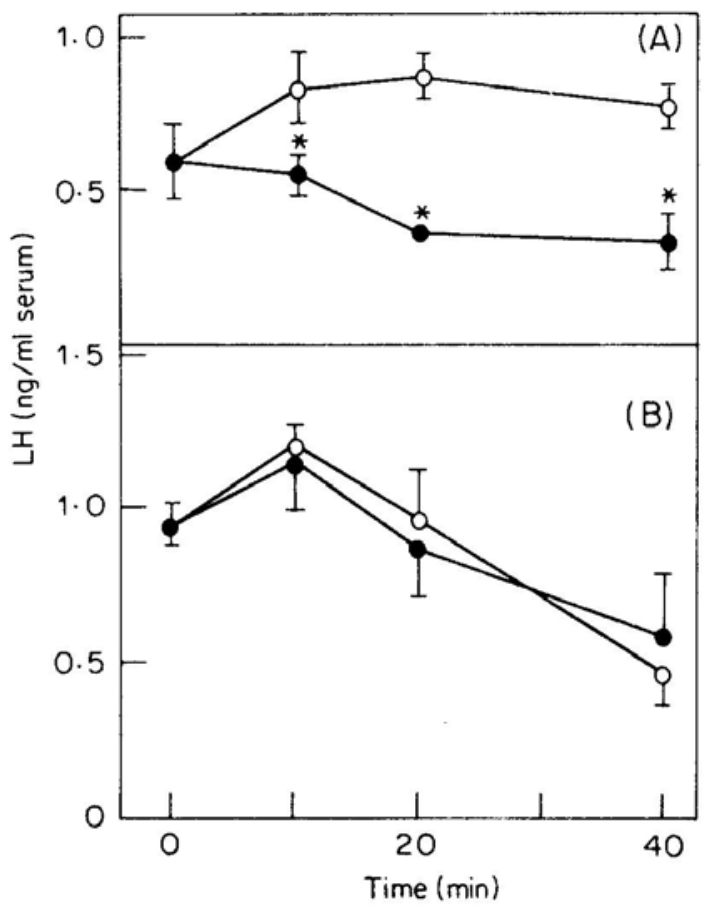

Figure 2. Effect of prolactin on pituitary response to LHRH during lactation in the presence (A) and absence of suckling stimulus (B). $P$ values were calculated for values at any given time between the treated and untreated groups. All values $>0.05$ were considered to be not significant.

* $P<0.02(\circ)$, No Prolactin; $(\bullet)$, plus prolactin.

actively suckling group: $0.6 \pm 0.12 \mathrm{ng} \mathrm{hLH} / \mathrm{ml} P<0 \cdot 001$ ), they take a longer time to show maximal increase $(20 \mathrm{~min})$ and the levels were maintained at this level for $40 \mathrm{~min}$ (figure 2A). The difference between the control lactating $v s$ actively suckling lactating group was more evident when $5 \mathrm{mg}$ of ovine prolactin (NIH PS5) dissolved in $0.5 \mathrm{ml}$ of $0.9 \% \mathrm{NaCl}$ was injected i.v. $1 \mathrm{~h}$ prior to LHRH injection. Prolactin injection significantly inhibited $(P<0.05)$ the responsiveness of the actively suckling lactating monkey to exogenous LHRH (figure 2A). In an independent study when responsiveness of actively lactating monkeys on different days of lactation (on day 15, 45, 75, 105 and 145 days of lactation) to $100 \mu \mathrm{g}$ of LHRH was compared it was observed that between days 45 to 105 the increase in LH was only minimal (increase by 20-30\%). In contrast on day 145 of lactation circulating LH levels could be increased by $400 \%$ within $20 \mathrm{~min}$ of injecting $100 \mu \mathrm{g}$ LHRH. These studies lead us to the conclusion that in addition to the neurogenic stimulus of suckling the circulating level of prolactin has an influence on the overall responsiveness of the pituitary to exogenous LHRH.

Effect of increasing endogenous prolactin levels on circulating LH levels in lactating monkeys

Since some of the above conclusions were based on the ability of exogenous prolactin to influence pituitary as well as gonadal responses it was felt worthwhile to determine: (a) a 
suitable method of increasing endogenous circulating levels of prolactin by drug treatment and (b) its effect on circulating LH levels in monkeys at different months of lactation.

The relative ability of $1 \mathrm{mg}$ of chlorpromazine injected by i.v. and i.m. route to increase endogenous prolactin levels is shown in figure 3A. Prolactin was measured by RIA in serum samples obtained at different periods following the drug administration. To achieve a response equivalent to the i.v. injection 5 times the dose $(5 \mathrm{mg})$ had to be given by i.m. (figure 3B). Interestingly administration of the drug by intranasal route using a method standardized by Anand Kumar et al. (1977) showed that the drug dosage could be markedly reduced $(\times 4$ less than the i.v. and $\times 20$ less than the i.m. dose) without a loss in its ability to significantly increase prolactin levels (figure 4 ). The ability of the drug, when administered by intranasal route to modulate LH levels also was checked by administering ovariectomized monkeys 250 and $500 \mu \mathrm{g}$ of chlorpromazine. It is evident from figure 5 that the drug had no effect on the high LH levels seen in the ovariectomized monkeys.
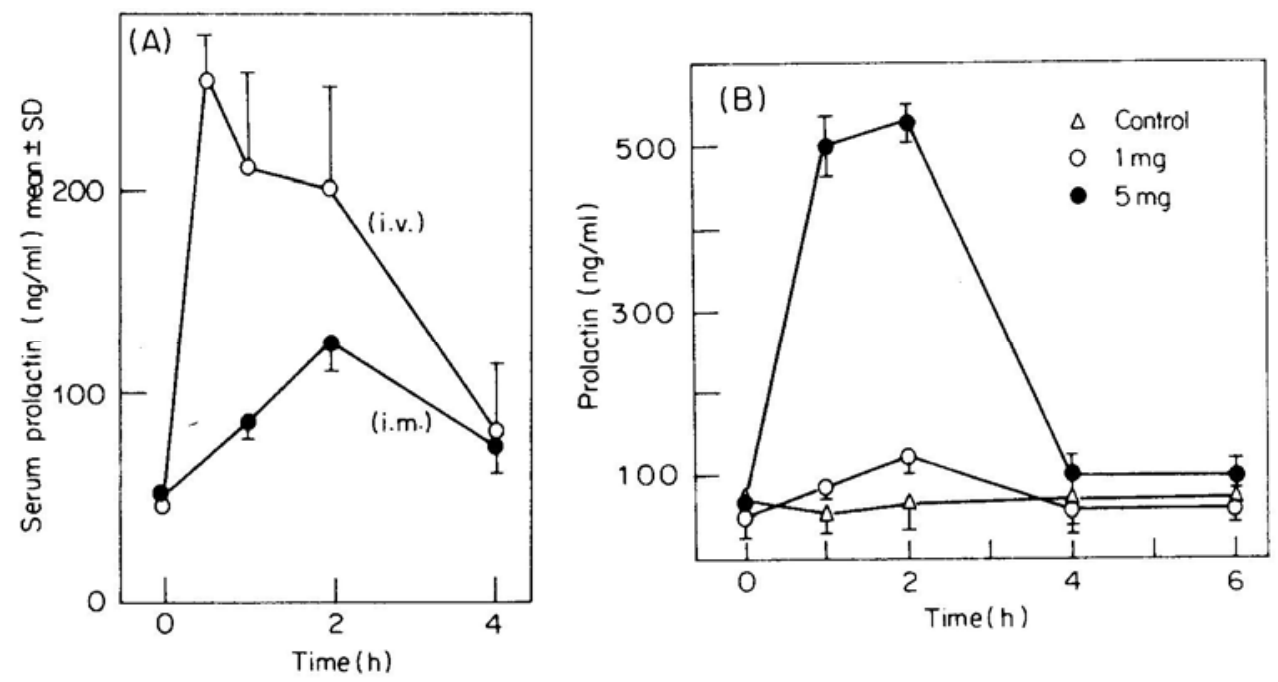

Figure 3. Establishing the dose and route of administration of chlorpromazine to increase serum prolactin levels.

A. Effect of injecting chlorpromazine (1 $\mathrm{mg} / \mathrm{monkey)}$ by i.m. and i.v. routes on serum prolactin levels ( $n$ of each group 3).

B. Effect of injecting different doses of chlorpromazine by i.m. route on serum prolactin levels of monkeys.

Assuming that monkeys during active lactation phase also would be going through 'silent abberant cycles' of approximately 28 days duration, groups of lactating monkeys ( $n$ per group was 3 ) in the 3 rd and 4th and 5th month of lactation were administered daily at the same hour between 10-14th day of cycle, $250 \mu \mathrm{g}$ of chlorpromazine by nasal spray. The control group received instead of the drug only the vehicle. The day of parturition was reckoned as day 1 of the first cycle and each cycle thereon was assumed 


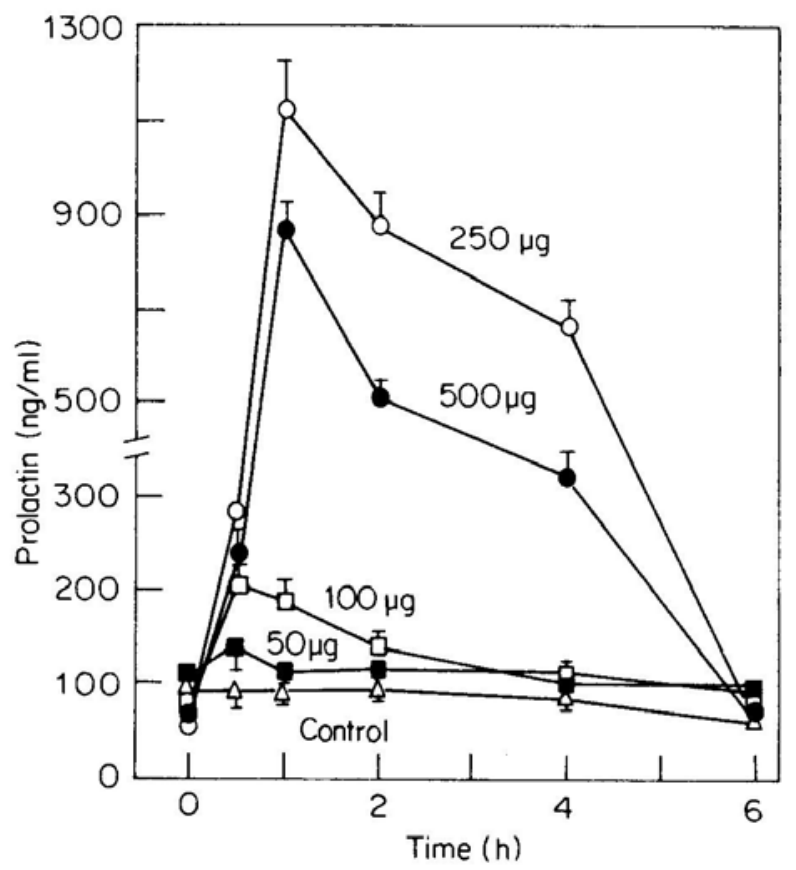

Figure 4. Effect of intranasal administration of chlorpromazine on serum prolactin levels. The drug was given in $10 \mathrm{sec}$ sprays up each nostril to unanesthetized monkeys according to the procedure of Anand Kumar et al., (1977). $n$ of each group was 3. The control monkeys received the vehicle (ethanol: propyleneglycol: water, 3:3:4) only. The efficiency of the nasal spray device was $1 \cdot 3 \%$.

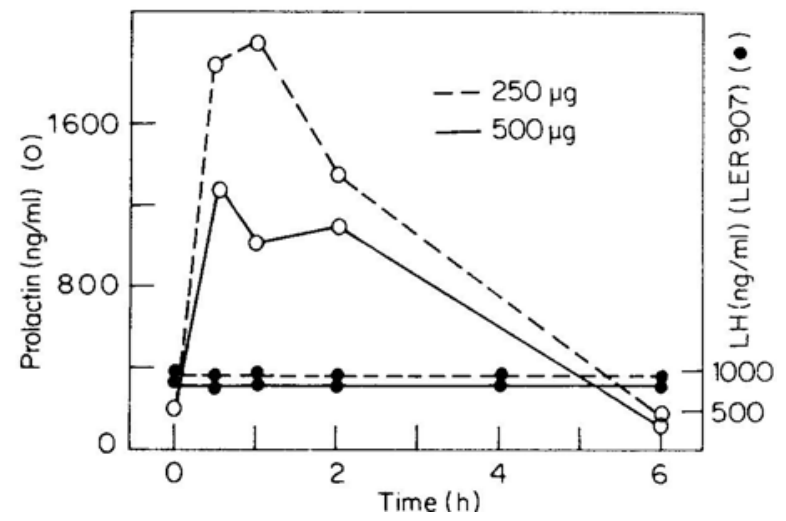

Figure 5. Relative effect of chlorpromazine nasal spray on prolactin and LH levels of ovariectomized monkeys-establishment of specificity of effect. Values are mean of 2 monkeys. Other procedures are similar to that described in figure 4.

to be of 28 days duration. Blood samples removed on each of the 5 days before nasal spray were assayed for prolactin and LH using appropriate RIAs standardized in the laboratory (Ramasharma et al., 1978). The results presented in figure 6 are mean of 5 samples taken from 3 monkeys each month in the control and treated group. It is 


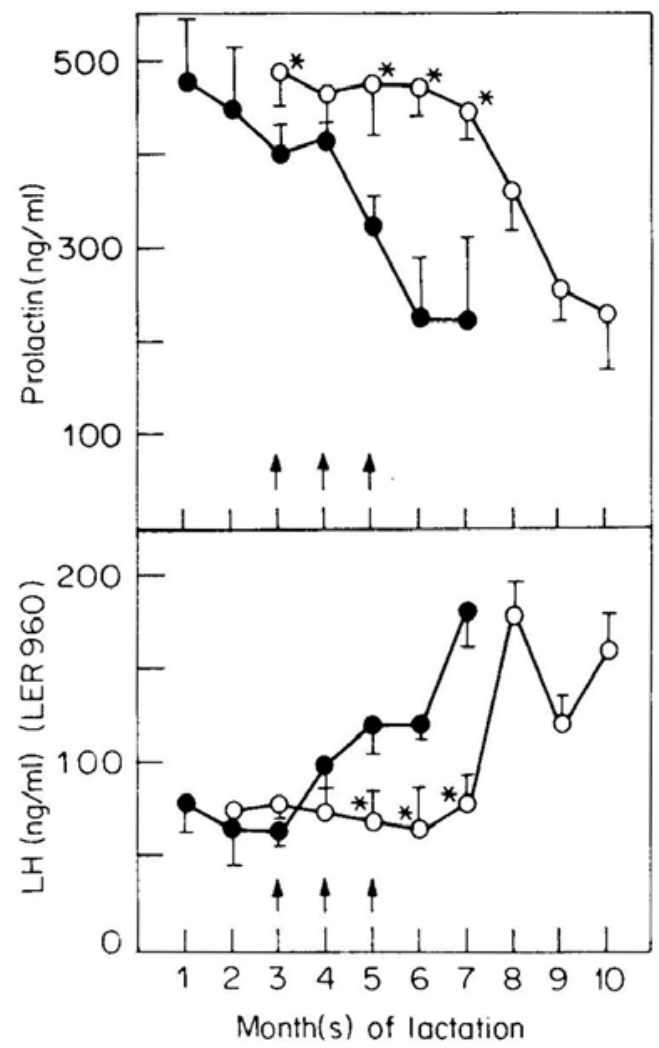

Figure 6. Effect of administering chlorpromazine by nasal spray $250 \mu \mathrm{g} /$ day/monkey between days 10-14 of each of 28 day 'silent cycles' (see text for explanation) starting from the 3rd month of lactation on serum prolactin and LH levels. The values reported are mean of 5 samples collected each day before the spray from 3 monkeys in each group. (•), Control; (०), drug treated.

* Significantly different from appropriate controls $P<0 \cdot 05$.

evident that in the control group prolactin levels decreased markedly by the 5 th month of lactation and by this time LH levels of the controls also showed a marked elevation. In the drug treated group, however, the decrease in prolactin by significant amounts occurred only by the 8th month and this coincided with the occurrence in LH levels in that month in surge amounts. It should be noted that by this time despite the drug induced high prolactin levels, due to reduced suckling (as the baby starts sharing more and more of the solid food of the mother) the LH levels tend to increase.

Effect of increasing serum prolactin levels on the return to cyclicity of lactating bonnet monkeys

It is evident from the results presented in table 1 that increasing serum prolactin levels either by exogenous ovine prolactin injection or by drug treatment leads to a significant postponement (by 3 months) in the onset of first postparturient menses. It is apparently not possible to postpone the occurrence of menses any further due to marked reduction 
Table 1. Effect of increasing serum prolactin levels on the return to cyclicity of lactating bonnet monkeys.

\begin{tabular}{|c|c|c|}
\hline Treatment & $\begin{array}{c}\text { Means of } \\
\text { administration }\end{array}$ & $\begin{array}{c}\text { Onset of 1st menses } \\
\text { following parturition } \\
\text { days } \pm \text { SD }\end{array}$ \\
\hline $\begin{array}{l}\text { Control (6) } \\
\text { Ovine Prolactin }\end{array}$ & Injection/intra-nasal & $218 \pm 4 \cdot 4$ \\
\hline $\begin{array}{l}2 \mathrm{mg} \text { /injection } \\
\text { Chlorpromazine }\end{array}$ & s.c. & $303^{*} \pm 11$ \\
\hline $\begin{array}{l}5 \mathrm{mg} / \text { injection } \\
\text { Chlorpromazine }\end{array}$ & i.m. & $312^{*} \pm 24$ \\
\hline $250 \mu \mathrm{g} / \mathrm{spray}$ & Intra-nasal & $316^{*} \pm 9 \cdot 1$ \\
\hline
\end{tabular}

Vehicle or drug was given by injection or nasal spray on days 10-14 of each month (of 28 day duration) beginning the 3rd month of lactation. Numbers in parenthesis indicate $\mathrm{N}$.

* Significantly different from the controls $P<0 \cdot 001$.

in the suckling stimulus. Administration of as low as $250 \mu \mathrm{g}$ of chlorpromazine per day for 5 days per cycle by nasal spray seems to be adequate to achieve the desired goal.

\section{Conclusion}

It appears from the foregoing that while the ovary of the lactating bonnet monkey is capable of responding to exogenous gonadotropin stimulus, its responsiveness in terms of estrogen production can be severely curtailed by exogenous prolactin treatment. A similar observation was made earlier using the lactating rat as a model system (Maneckjee et al., 1977). The mechanism by which excess prolactin inhibits the ability of the ovary to produce estrogen in response to exogenous gonadotropin is yet to be unraveled. Prolactin also appeared to influence the sensitivity of the pituitary to exogenous LHRH. The lactating state is exemplified by the presence of two types of stimuli, the neurogenic stimulus of suckling and high prolactin levels. It appears that high prolactin levels in itself, in the absence of 'suckling stimulus' is unable to influence pituitary responsiveness to exogenous LHRH. This perhaps is the reason why chlorpromazine treatment every month starting from the 3rd month of lactation was not able to postpone the onset of first post-parturient menses indefinitely, the day of $1 \mathrm{st}$ menses could be postponed by 3 months only (from 218 to 312 days). Endogenous prolactin level has been increased in the present series of experiments by giving the drug chlorpromazine. Interestingly the dosage of the drug could be markedly reduced (by $\times 20)$ if it was delivered in the nasal spray mode. At this low level $(250 \mu \mathrm{g})$, the drug did not induce sleep in the receipient monkeys. Whether this type of drug therapy can be used in lactating women to extend the state of lactational amennorhea is perhaps worth investigating. It is possible that other drugs effecting central nervous system can also be delivered by the intranasal mode. We have observed for instance, that the effective dose of ergobromocryptin, a drug reducing prolactin levels can be significantly reduced by giving it by the intranasal mode. 


\section{Acknowledgements}

Financial aid from the Indian Council of Medical Research, New Delhi and the WHO Small Supplies Programme, Geneva is gratefully acknowledged. The help rendered by Mr. B. Shanmugavelu in this study is appreciated.

\section{References}

Amenomori, Y., Chen, C. L. and Meites, J. (1970) Endocrinology, 86, 506.

Anand Kumar, T. C., David, G. F. X. and Puri, V. (1977) Nature, (London), 270, 532.

Maneckjee, R. and Moudgal, N. R. (1975) J. Reprod. Fert., 43, 33.

Maneckjee, R., Moudgal, N. R. and Srinath, B. R. (1977) Fert. Steril., 28, 986.

Maneckjee, R., Srinath, B. R. and Moudgal, N. R. (1976) Nature (London), 262, 507.

Mukku, V. R. and Moudgal, N. R. (1975) Endocrinology, 97, 1455.

Muralidhar, K., Maneckjee, R. and Moudgal, N. R. (1977) Endocrinology, 100, 1137.

Murthy, G. S. R. C., Ramasharma, K., Mukku, V. R., Srinath, B. R. and Moudgal, N. R. (1980) in Non-human primate models for study of human reproduction, (ed. T. C. Anand Kumar), (Karger Basel), p. 50.

Ramasharma, K., Muralidhar, K. and Moudgal, N. R. (1978) Indian J. Exp. Biol., 16, 153.

Srinath, B. R. (1980) in Non-human primate models for study of human reproduction (ed. T. C. Anand Kumar) (Karger Basel), p. 17.

Trekle, J., Blake, C. A. and Sawyer, C. H. (1972) Endocrinology, 91, 49. 